\title{
New parameters and transit timing studies for OGLE2-TR-L9 $b^{\star}$
}

\author{
M. Lendl ${ }^{1,3,5}$, C. Afonso ${ }^{1}$, J. Koppenhoefer ${ }^{2,6}$, N. Nikolov ${ }^{1}$, Th. Henning ${ }^{1}$, M. Swain ${ }^{4}$, and J. Greiner ${ }^{6}$ \\ 1 Max Planck Institute for Astronomy, Königstuhl 17, 69117 Heidelberg, Germany \\ e-mail: Monika. Lendl@unige.ch \\ 2 Universitäts-Sternwarte München, Scheinerstr. 1, 81679 Munich, Germany \\ 3 Institut für Astronomie, Universität Wien, Türkenschanzstr. 17, 1180 Wien, Austria \\ 4 Jet Propulsion Laboratory, California Institute of Technology, 4800 Oak Grove Drive, Pasadena, California 91109-8099, USA \\ Observatoire de Genève, Université de Genève, 51 chemin des Maillettes, 1290 Versoix, Switzerland \\ 6 Max Planck Institute for Extraterrestrial Physics, Giessenbachstr., 85748 Garching, Germany
}

Received 5 May 2010 / Accepted 22 June 2010

ABSTRACT

\begin{abstract}
Context. Repeated observations of exoplanet transits allow us to refine the planetary parameters and probe them for any time dependent variations. In particular deviations of the period from a strictly linear ephemeris, transit timing variations (TTVs), can indicate the presence of additional bodies in the planetary system.

Aims. Our goal was to reexamine the largely unstudied OGLE2-TR-L9 system with high cadence, multi-color photometry in order to refine the planetary parameters and probe the system for TTVs.

Methods. We observed five full transits of OGLE2-TR-L9 with the GROND instrument at the ESO/MPG $2.2 \mathrm{~m}$ telescope at La Silla Observatory. GROND is a multichannel imager that allowed us to gather simultaneous light curves in the $g^{\prime}, r^{\prime}, i^{\prime}$, and $z^{\prime}$ filters.

Results. From our analysis we find that the semi-major axis and the inclination differ from the previously published values. With the newly observed transits, we were able to refine the ephemeris to $2454492.80008( \pm 0.00014)+2.48553417\left( \pm 6.4 \times 10^{-7}\right) E$. The newly derived parameters are $a=0.0418 \pm 0.0015 \mathrm{AU}, r_{\mathrm{p}}=1.67 \pm 0.05 R_{j}$, and inc $=82.47^{\circ} \pm 0.12$, differing significantly in $a$ and inc from the previously published values. Within our data, we find indications for TTVs.
\end{abstract}

Key words. techniques: photometric - planetary systems - planets and satellites: individual: OGLE2-TR-L9 b

\section{Introduction}

In the study of extrasolar planets, the observation of planetary transits has a prominent position. It allows us to determine several parameters that are not accessible by other means, shedding more light on the nature of the transiting planet. From the transit light curve itself, the planetary radius and the orbital inclination can be found by investigating its shape and depth. Together with radial velocity measurements, the knowledge of the inclination allows us to determine the true mass of the planet, which can then be used in conjunction with the radius to find the planet's mean density, allowing its composition to be constrained. With spectroscopic observations during transit and secondary eclipse, the chemical composition of planetary atmospheres can be studied (e.g. Charbonneau et al. 2002; Swain et al. 2008).

Despite its advantages and increasing success, the transit method presents limitations for small and farther out planets: it is strongly biased towards close-in planets since the geometrical probability of a planet to transit decreases with orbital separation and the transit signal becomes much less distinct for small planets, reaching down to a transit depth of only $0.01 \%$ for an Earth-sized planet orbiting a solar-type star. With increasing orbital periods, scheduling becomes a major constraint on the discovery of planets, as transit signals become sparser in time, and long-term observations are required.

* Photometric data are only available in electronic form at the CDS via anonymous ftp to cdsarc.u-strasbg.fr (130.79.128.5) or via

http://cdsweb.u-strasbg.fr/cgi-bin/qcat?J/A+A/522/A29
The transit timing method has the potential to detect small transiting or non transiting objects such as moons, Trojans, or additional planets that can be difficult to find with current methods. It makes use of the fact that the gravitational impact of these objects can be measured as dynamical interactions within the system, causing the time between successive transits to vary, and in turn the transit occurs earlier or later than expected (Holman \& Murray 2005). The transit timing method aims at discovering additional objects by searching for changes in the mid-transit times of close-in transiting exoplanets. Naturally, this technique is more sensitive to massive perturbers but it can be sensitive enough to detect planets of only a few Earth masses which are located near mean motion resonances with the transiting giant. Several searches for TTVs in exoplanetary systems have been undertaken up to now (e.g. Alonso et al. 2009; Díaz et al. 2008; Holman et al. 2006). While no additional objects have been found with this technique, it has been used to pose limits on the existence of such objects in several cases (e.g. Rabus et al. 2009; Adams et al. 2010). To detect TTVs one needs to observe a large number of transits and build up a database of mid-transit times covering several seasons. Additionally, the repeated observation of transits enables us to refine the planetary parameters.

The subject of this paper, OGLE2-TR-L9 b, has been identified as a planetary candidate in the publicly available data of the OGLE-II project (Udalski et al. 1997) by Snellen et al. (2007). It was recently confirmed as a transiting exoplanet with the observation of a full transit and radial velocity measurements by Snellen et al. (2009). OGLE2-TR-L9 b has a mass of $4.5 \pm 1.5 M_{J}$ and a period of approximately 2.5 days (Snellen et al. 2009) 
orbiting an F3V star, at this point in time the hottest star known to host a transiting planet.

In this paper, we present five new transits of OGLE2TR-L9 observed with the GROND instrument (Greiner et al. 2008) mounted at the ESO/MPG $2.2 \mathrm{~m}$ telescope at La Silla Observatory during April and May 2009. We recorded each transit simultaneously in four optical channels, $g^{\prime}, r^{\prime}, i^{\prime}$, and $z^{\prime}$. Using this newly available data and reanalyzing the data obtained by Snellen et al. (2009), we redefine the planetary parameters and conduct a first search for TTVs. An overview of the observations and the data reduction methods used will be given in Sect. 2, while a description of the data analysis and error estimation is presented in Sect. 3. Section 4 summarizes the obtained results.

\section{Observations and data reduction}

\subsection{Observations}

We observed five transits of OGLE2-TR-L9 using the GROND (Gamma Ray Burst Optical and Near-Infrared Detector) instrument (Greiner et al. 2008) mounted at the ESO/MPG $2.2 \mathrm{~m}$ telescope at La Silla Observatory. Designed with the aim of observing gamma-ray-burst afterglows, GROND simultaneously observes in 4 optical $\left(\begin{array}{llll}g^{\prime} & r^{\prime} & i^{\prime} & z^{\prime}\end{array}\right)$ and 3 infrared $(J H K)$ channels allowing us to gather 4 optical light curves of each transit event. In the infrared channels, series of exposures of $10 \mathrm{~s}$ each were obtained. Since dithering was switched off for a large part of the observations to improve time sampling and photometric precision, no stacking of the infrared frames is possible with the standard way of background subtraction. We therefore ignore the $J H K$ channels in our analysis.

The observations took place during the nights of April 10, 15, 20, 25 and May 15 2009, corresponding to the Epochs 177, 179, 181, 183 and 191 based on the ephemeris given by Snellen et al. (2009). During each night, we observed the full transit plus at least $20 \mathrm{~min}$ of baseline before ingress and after egress. For all observations, the exposure time was kept fixed at $46.4 \mathrm{~s}$. During the night of April 10, we used the slow ( $\sim 5 \mathrm{~s}$ ) readout mode achieving a cadence of $2.05 \mathrm{~min}$. We incurred some problems with guiding during the night of April 15, leading to unstable data quality. During this night, we were able to test the fast $(\sim 15 \mathrm{~s})$ readout mode and observed with a cadence of $1.95 \mathrm{~min}$. For the nights of April 20, 25 and May 15, we used the fast readout mode, increasing the cadence to $1.31 \mathrm{~min}$ for April 20 and $1.25 \mathrm{~min}$ for April 25 and May 15.

In our analysis, we included the transit observed with GROND on January 27, 2008 by Snellen et al. (2009). The observation procedure was essentially the same as in our first observation (April 10). A detailed description of the observations can be found in Snellen et al. (2009), while the data reduction was redone for this work as described below.

\subsection{Data reduction}

With one of the goals of the initial OGLE observations being the detection of micro lensing events, OGLE2-TR-L9 is located in a rather crowded field. Fortunately, OGLE2-TR-L9 is reasonably bright $(I=13.97 \mathrm{mag})$ and fairly isolated, with only very minor sources in its vicinity allowing the use of aperture photometry.

The image calibration was performed with the mupipe software which has been developed at the University Observatory

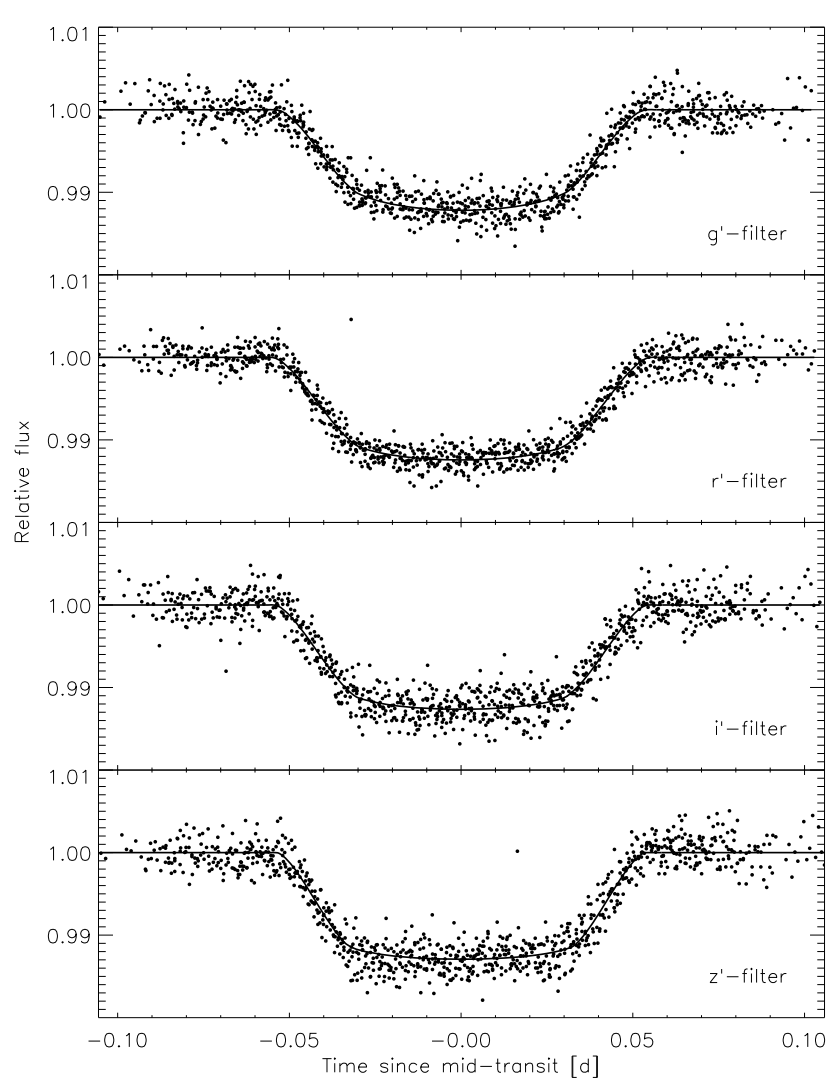

Fig. 1. The phased light-curves of OGLE2-TR-L9. From top to bottom, the filters are: $g^{\prime}, r^{\prime}, i^{\prime}$, and $z^{\prime}$. The phased light curves also contain the new reduction of the data from Snellen (2009). The best fit models are depicted as continuous lines.

Munich $^{1}$, and encompassed overscan and flatfield corrections. Then we carried out flux measurements for aperture photometry using IRAF ${ }^{2}$ daophot procedures. We iteratively selected approximately 15 of the most stable stars in the field (with the exact number depending on filter and seeing conditions) and combined them to a reference source to be used for differential photometry.

For comparison, we also performed aperture photometry using mupipe but found that the light curves produced with IRAF show a smaller scatter. Therefore we only used the light curves produced with IRAF for the analysis. Comparing the light curves in the four optical channels, we found that the $r^{\prime}$ filter shows the best accuracy, with a photometric accuracy of $1.5 \mathrm{mmag}$, while we achieved $1.8 \mathrm{mmag}$ for the $g^{\prime}$ channel, and $2.0 \mathrm{mmag}$ for the $i^{\prime}$ and $z^{\prime}$ channels. We present all light curves in Fig. 6.

\section{Determination of system parameters}

The light curve fits were performed using the model for a planetary transit given by Mandel \& Agol (2002). We assumed a quadratic limb darkening law and chose the coefficients according to Claret (2004) for a star with metallicity $[\mathrm{Fe} / \mathrm{H}]=0.0$,

\footnotetext{
1 Mupipe is available from http://wwW.usm.uni-muenchen.de/ people/arri/mupipe/.

2 IRAF is distributed by the National Optical Astronomy Observatories, which are operated by the Association of Universities for Research in Astronomy, Inc., under cooperative agreement with the National Science Foundation.
} 
Table 1. The results for the planetary to stellar radius ratio, the semimajor axis in units of the stellar radius, and the inclination for the filters used next to the final parameters produced by a combined fit of all available light curves.

\begin{tabular}{llllll}
\hline \hline Filter: & $g^{\prime}$ & $r^{\prime}$ & $i^{\prime}$ & $z^{\prime}$ & All \\
\hline$r_{\mathrm{p}} / r_{*}$ & 0.11021 & 0.1116 & 0.1121 & 0.1123 & 0.1120 \\
& \pm 0.0009 & \pm 0.0006 & \pm 0.0006 & \pm 0.0006 & 0.003 \\
$a / r_{*}$ & 5.99 & 5.80 & 5.93 & 6.23 & 5.88 \\
& \pm 0.15 & \pm 0.11 & \pm 0.11 & \pm 0.13 & \pm 0.06 \\
inc $\left[^{\circ}\right]$ & 82.69 & 82.31 & 82.56 & 83.25 & 82.47 \\
& \pm 0.32 & \pm 0.25 & \pm 0.24 & \pm 0.26 & \pm 0.12 \\
\hline
\end{tabular}

surface gravity $\log (g)=4.5$ and effective temperature $T_{\text {eff }}=$ $7000 \mathrm{~K}$. We found the best fit to our data by minimizing the $\chi^{2}$ using a downhill simplex algorithm as implemented in the AMOEBA code (Press et al. 1992). Our goal was to find the best-fitting values for the central transit time $t_{\mathrm{c}}$, the ratio of the planetary and stellar radii $r_{\mathrm{p}} / r_{*}$, the planetary semi-major axis in units of the stellar radius $a / r_{*}$, and the orbital inclination inc. The above parameters have the advantage that they can be derived directly from the light curve without assumptions regarding stellar properties other than limb darkening. As starting values, we assumed the central transit times calculated according to the ephemeris given by Snellen et al. (2009), and typical values of planetary transits for the other parameters: $r_{\mathrm{p}} / r_{*}=0.1$, $a / r_{*}=20$ and inc $=90^{\circ}$. After the best parameters were found for each light curve, we used the resulting mid-transit times to phase fold the data producing the light curves shown in Fig. 1. To improve on the parameters derived from the data in each of the four filters, these phase folded light curves were again fitted for $r_{\mathrm{p}} / r_{*}, a / r_{*}$ and inc. The final values were found by a combined fit of all available light curves. The resulting parameters are shown together with the results for the phased light curves in Table 1 and the corresponding model is depicted as a continuous line in Fig. 1.

To estimate the errors of the determined parameters, we used the Bootstrap Monte-Carlo Method (Press et al. 1992) which works by creating a large number of representations of the data by randomly choosing, with replacement, subsets from the original data set. This means that each newly created data set contains the same number of points as the original data set but with some points left out and some points duplicated. For each bootstrap data set, the best fitting parameters are found using a procedure identical to the analysis of original data and the errors are calculated from the distribution of the results. To get a second estimate for the errors in the central transit time, we kept the model parameters fixed at their best values and let $t_{\mathrm{c}}$ vary until the deviation in the $\chi^{2}$ exceeded $\Delta \chi^{2}=1$. The resulting errors are in good agreement with the errors derived from the Bootstrap Monte-Carlo Method and range from $29 \mathrm{~s}$ for transits observed with a lower cadence to $16 \mathrm{~s}$ for transits observed with higher cadence. Here, it should be pointed out that the above error treatment does not incorporate the contribution of correlated low frequency noise (Pont et al. 2006). Thus, the errors on the mid-transit points are likely to be underestimated.

\section{Results}

\subsection{Planetary parameters}

In Table 1, we present the results for the phased $g^{\prime}, r^{\prime}, i^{\prime}$, and $z^{\prime}$ light curves. As can be seen in Fig. 2, the values for the planetary radius mainly agree within one sigma, apart from the

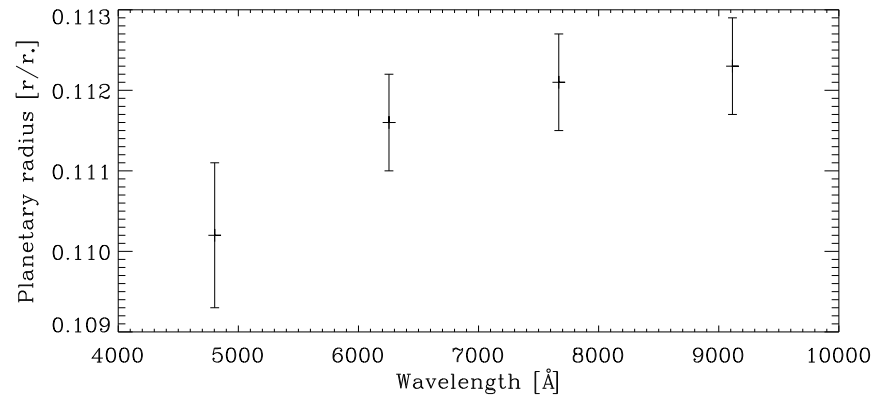

Fig. 2. The results for the radius ratio $r_{\mathrm{p}} / r_{*}$ obtained from the phased light curves against the filter wavelengths. The values show a slight trend towards smaller planetary radii with decreasing wavelength.
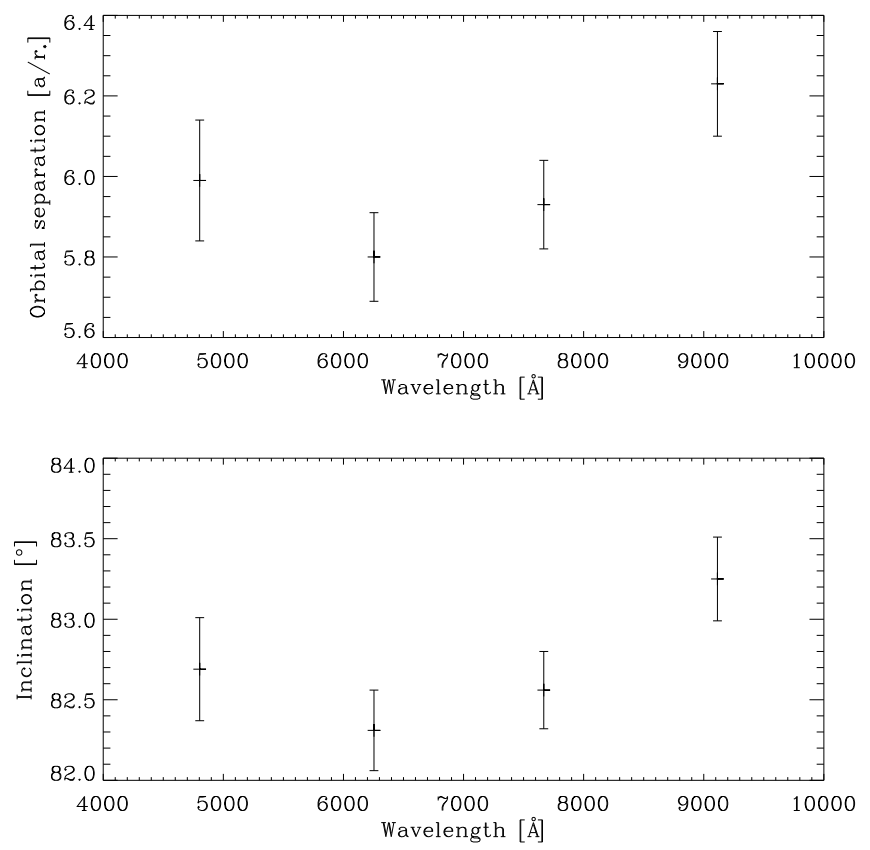

Fig. 3. The results for the planetary semi-major axis (upper panel) and the inclination (lower panel) against the filter wavelengths. The fact that the shapes of the variations are very similar shows the degeneracy present between the semi-major axis and the inclination: larger solutions for the planetary separation favor higher inclinations.

Table 2. The final results for the planetary parameters derived from the data presented in this work.

\begin{tabular}{ll}
\hline \hline Parameter & \\
\hline Semi-major axis [AU] & $0.0418 \pm 0.0015$ \\
Planetary radius $\left[R_{\text {jup }}\right]$ & $1.67 \pm 0.05$ \\
Inclination $\left[{ }^{\circ}\right]$ & $82.47 \pm 0.12$ \\
Transit duration [d] & $0.1092 \pm 0.0021$ \\
Period [d] & $2.48553417 \pm 6.4 \times 10^{-7}$ \\
$T_{0}[\mathrm{HJD}]$ & $2454492.80008 \pm 0.00014$ \\
Stellar density $\left[\rho_{\odot}\right]$ & $0.442 \pm 0.014$ \\
\hline
\end{tabular}

value derived from the $g^{\prime}$ filter which lies slightly below. While values for the planetary semi-major axis and inclination found with the $g^{\prime}, r^{\prime}$ and $i^{\prime}$ photometry agree within one sigma, the values derived from the $z^{\prime}$ band observations favor a larger orbital separation combined with a higher inclination (see Fig. 3). 
Table 3. All mid-transit times of OGLE2-TR-L9 known up to date.

\begin{tabular}{ll}
\hline \hline$t_{\mathrm{c}}$ [HJD-2 400 000] & Reference \\
\hline $50478.661 \pm 0.0012$ & Snellen et al. (2007) \\
$54492.80086 \pm 0.00033$ & this work \\
$54932.73944 \pm 0.00031$ & this work \\
$54937.70976 \pm 0.00029$ & this work \\
$54942.68169 \pm 0.00022$ & this work \\
$54947.65232 \pm 0.00019$ & this work \\
$54967.53689 \pm 0.00022$ & this work \\
\hline
\end{tabular}

As pointed out by Seager \& Mallén-Ornelas (2003), the mean stellar density can be derived directly from the light curve shape and the planetary period. Following the description of Seager \& Mallén-Ornelas (2003), we find the stellar density to be $\rho_{*}=0.442 \pm 0.014\left[\rho_{\odot}\right]$, which is in good agreement with the previously published value.

To find the planetary parameters $a_{\mathrm{p}}$ and $r_{\mathrm{p}}$, we adopted the stellar parameters from Snellen et al. (2009) and combined them with the best-fit values obtained from our analysis. The results and their respective errors can be found in Table 2. All values are in good agreement with the values published in Snellen et al. (2009) except for $a_{\mathrm{p}}$ and inc where our new results are significantly different. The reason for this discrepancy is an error in the calculation of $a_{\mathrm{p}}$ in Snellen et al. (2009) where $1 / M_{*}$ was used instead of $M_{*}$ when transforming the measured period into a semi-major axis using Kepler's third Law. The error in semimajor axis propagated into a wrong inclination value. We recommend to use the values published here for future work because of this calculation error in Snellen et al. (2009) and because the new values are based on a significantly larger dataset.

\subsection{A new ephemeris}

To derive a new ephemeris for OGLE2-TR-L9 b, we used all six observed transits of OGLE2-TR-L9 together with an ephemeris derived from the OGLE-II dataset by Snellen et al. (2007). It should be noted that this "mid-transit point" is derived from an OGLE-II light curve which was constructed from 500 data points with irregular cadence collected over a period of 3.5 years. From our new analysis of the transit observed by Snellen et al. (2009) on January 27, 2008, we find the central transit time to be HJD $2454492.80086 \pm 0.00033$ instead of HJD $2454492.79765 \pm 0.00039$. All mid-transit times known for OGLE2-TR-L9 can be found in Table 3. Fitting a constant period to all available mid-transit times gives the following new ephemeris:

$$
\begin{aligned}
T_{\mathrm{c}}(E)[\mathrm{HJD}]= & 2454492.80008( \pm 0.00014) \\
& +2.48553417\left( \pm 6.4 \times 10^{-7}\right) E .
\end{aligned}
$$

\subsection{Transit timing and transit duration variations}

Finally, it is possible to search for TTVs. To do so, the observed minus calculated $(\mathrm{O}-\mathrm{C})$ values with reference to the ephemeris determined above are computed for each of the given mid-transit points. The result is shown in Fig. 4. Here epoch -1615 corresponds to the ephemeris derived by Snellen et al. (2007) from the OGLE-II data, epoch 0 corresponds to the corrected midtransit time from Snellen et al. (2009) and the last five epochs correspond to the newly observed transits. It can be seen that the transit times show some deviations from a constant period as the transit of epoch 0 occurs $1.5 \mathrm{~min}$ later and the transit of
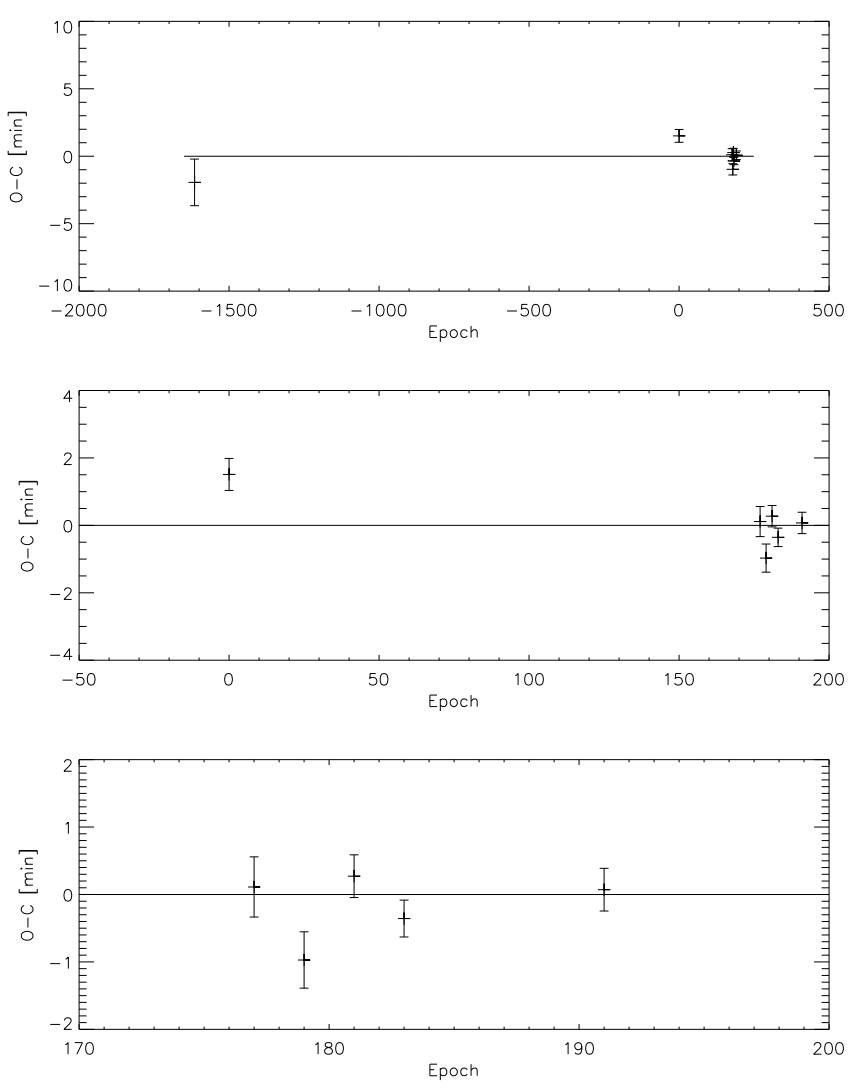

Fig. 4. The O-C diagram for the ephemeris calculated from all known mid-transit times of OGLE2-TR-L9 b. In the upper panel, all points are shown, while the lower two panels zoom in on the new points together with the point found from the transit in 2008 (middle panel) and solely the new points (lower panel). The mid-transit points of the epochs 0 and 179 occur $1.5 \mathrm{~min}$ later and $1.0 \mathrm{~min}$ earlier than expected, respectively.
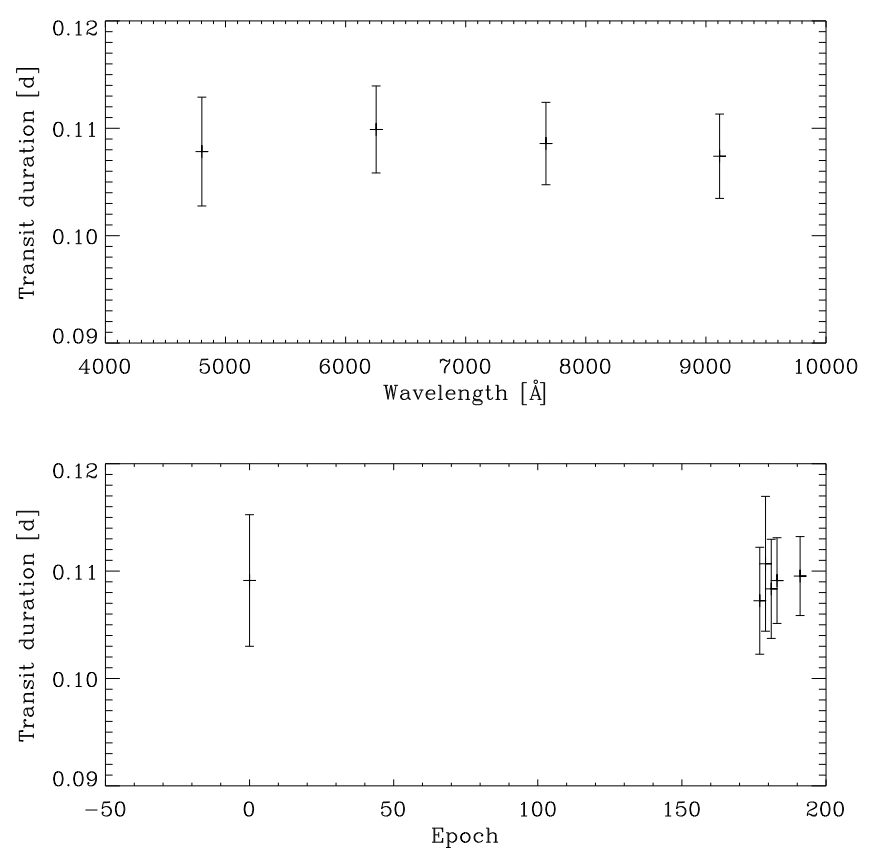

Fig. 5. The transit durations obtained from the phased light curves in the four filters (upper panel) and the light curves for each transit event (lower panel). All values agree within their errors. 
M. Lendl et al.: New parameters and transit timing studies for OGLE2-TR-L9 b

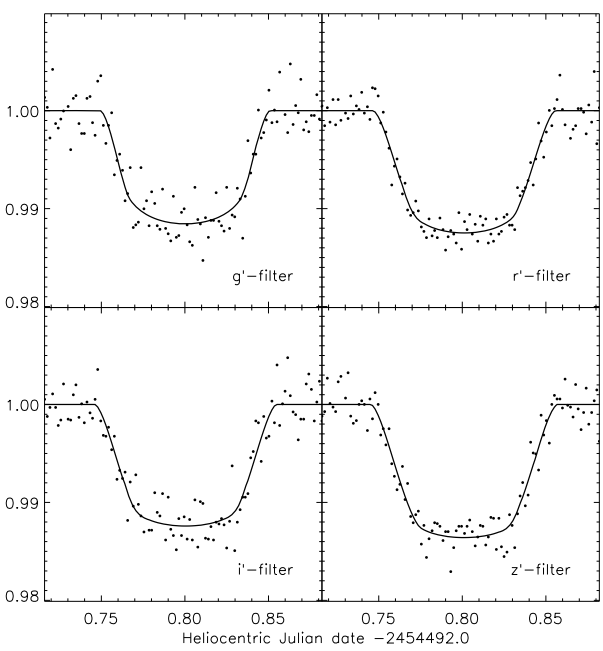

(a)

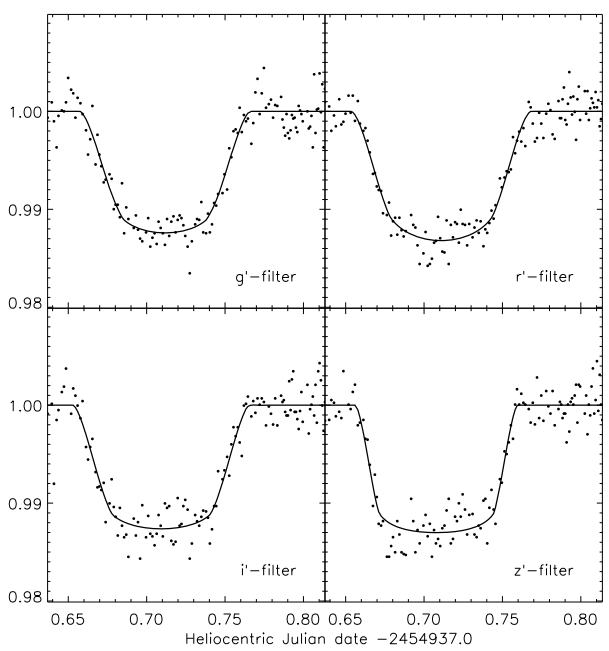

(c)

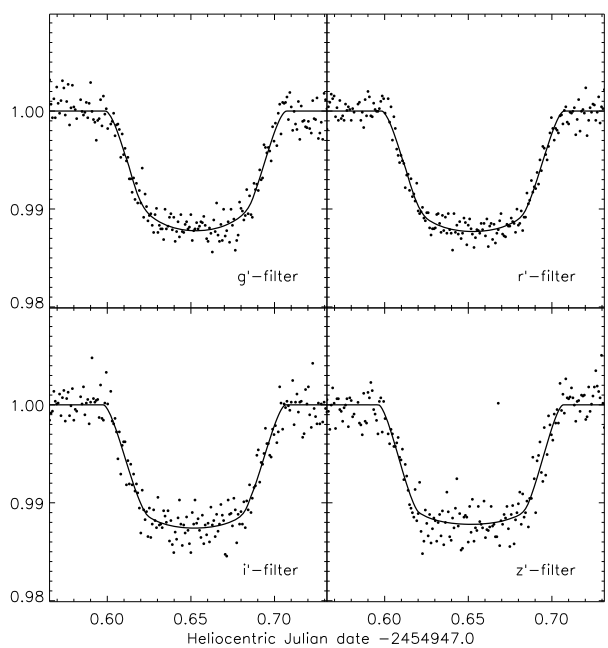

(e)

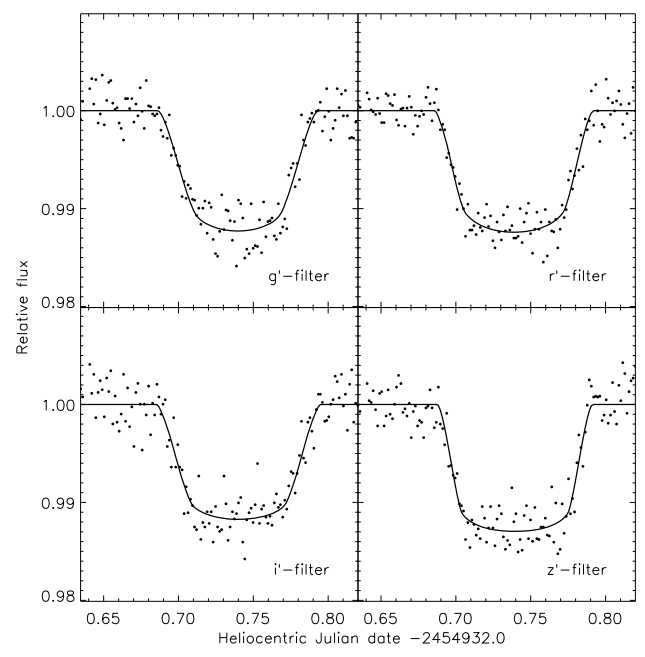

(b)

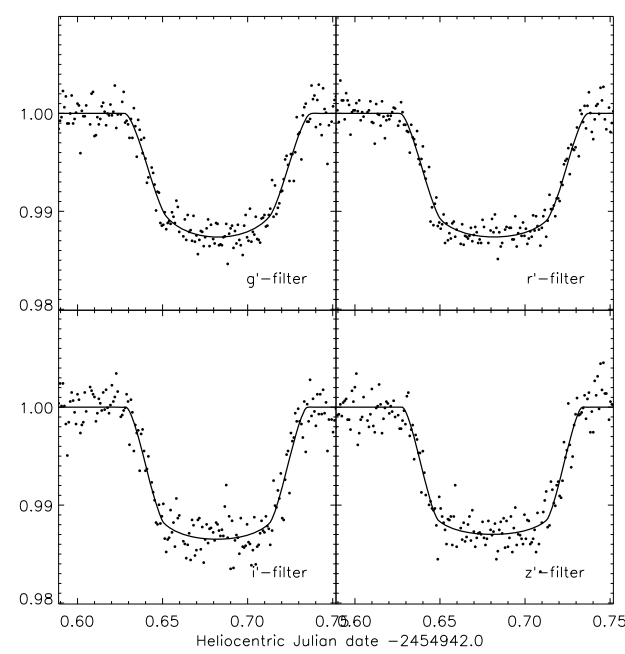

(d)

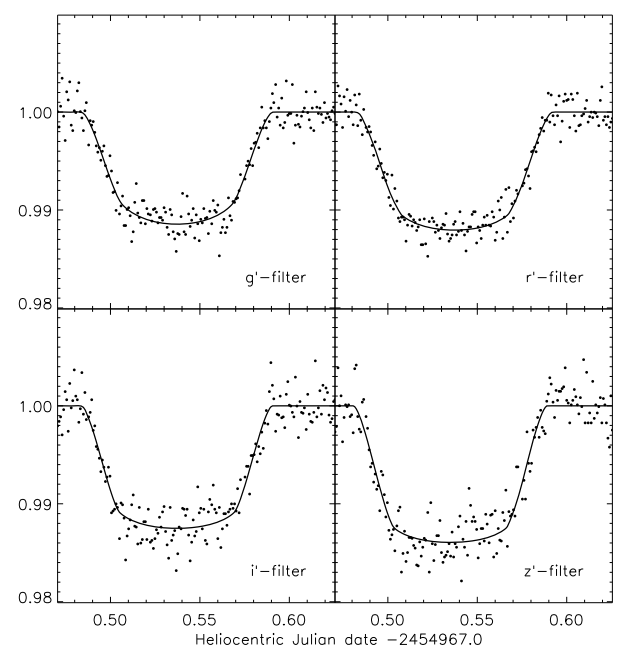

(f)

Fig. 6. All light curves in the four optical channels. The data were recorded during the nights of January 28, 2008 a) (by Snellen et al. 2009), April 10 b), April 15 c), April 20 d), April 25 e) 2009 and May 15 f) 2009. The continuous lines represent the best fit to the respective data. 
epoch 179 occurs 1.0 min earlier than expected. Fitting a constant period to the data as done in Sect. 4.2 gives $\chi_{\text {red }}^{2}=3.86$ yielding a confidence level of $>99.75 \%$. However, one must take into account that the errors on the central transit times are likely to be underestimated due to correlated noise (see Sect. 3).

Next to variations in the transit timing, we also searched our data for variations in the transit duration which could hint at the existence of exo-moons (Kipping 2009). For this purpose, we measured the transit durations based on the best models of both the phased light curves and the light curves derived from each transit event. The results are illustrated in Fig. 5 and show no detectable variation.

\section{Conclusion}

We have gathered five full transits of OGLE2-TR-L9 b with the GROND multichannel imager mounted on the ESO/MPG $2.2 \mathrm{~m}$ telescope at La Silla Observatory. With this new data, and including the transit observed by Snellen et al. (2009) in our analysis, we recalculated the parameters of OGLE2-TR-L9 $b$ and found different semi-major axis and inclination values compared to Snellen et al. (2009). This is due to a calculation error in the previous work and thus the values presented in this work should be used in the future. We studied the central transit times and transit durations for any variations that could be attributed to a perturbing body in the OGLE2-TR-L9 system. While the transit durations agree for all transits, the mid-transit points show indications of period variations.

Acknowledgements. We thank P. Afonso, F. Olivares and A. Rossi from the GROND team for their valuable support during the observations.
Based on observations collected at the European Organisation for Astronomical Research in the Southern Hemisphere, Chile (083.A-9005(A)) during MPG guaranteed time.

Part of the funding for GROND (both hardware as well as personnel) was generously granted from the Leibniz-Prize to Prof. G. Hasinger (DFG grant HA 1850/28-1).

\section{References}

Adams, E. R., López-Morales, M., Elliot, J. L., Seager, S., \& Osip, D. J. 2010, ApJ, 714, 13

Alonso, R., Aigrain, S., Pont, F., Mazeh, T., \& The CoRoT Exoplanet Science Team. 2009, in IAU Symp., 253, 91

Charbonneau, D., Brown, T. M., Noyes, R. W., \& Gilliland, R. L. 2002, ApJ, 568,377

Claret, A. 2004, VizieR Online Data Catalog, 342, 81001

Díaz, R. F., Rojo, P., Melita, M., et al. 2008, ApJ, 682, L49

Greiner, J., Bornemann, W., Clemens, C., et al. 2008, PASP, 120, 405

Holman, M. J., \& Murray, N. W. 2005, Science, 307, 1288

Holman, M. J., Winn, J. N., Latham, D. W., et al. 2006, ApJ, 652, 1715

Kipping, D. M. 2009, MNRAS, 392, 181

Mandel, K., \& Agol, E. 2002, ApJ, 580, L171

Pont, F., Zucker, S., \& Queloz, D. 2006, MNRAS, 373, 231

Press, W. H., Teukolsky, S. A., Vetterling, W. T., \& Flannery, B. P. 1992, Numerical recipes in C. The art of scientific computing, ed. S. A. Teukolsky, W. T. Vetterling, B. P. Flannery, \& W. H. Press

Rabus, M., Deeg, H. J., Alonso, R., Belmonte, J. A., \& Almenara, J. M. 2009, A\&A, 508, 1011

Seager, S., \& Mallén-Ornelas, G. 2003, ApJ, 585, 1038

Snellen, I. A. G., van der Burg, R. F. J., de Hoon, M. D. J., \& Vuijsje, F. N. 2007, A\&A, 476, 1357

Snellen, I. A. G., Koppenhoefer, J., van der Burg, R. F. J., et al. 2009, A\&A, 497, 545

Swain, M. R., Vasisht, G., \& Tinetti, G. 2008, Nature, 452, 329

Udalski, A., Kubiak, M., \& Szymanski, M. 1997, Acta Astron., 47, 319 